\title{
Poema de Chile, sus manuscritos y la valoración del legado de Gabriela Mistral*
}

\author{
Poem of Chile, its manuscripts and an assessment of the value of the \\ Gabriela Mistral legacy
}

\begin{abstract}
Soledad Falabella Luco ${ }^{1}$, Bernardita Domange ${ }^{2}$
${ }^{1}$ Universidad Diego Portales, Facultad de Comunicación y Letras, Santiago, Chile; Universidad de Chile, Facultad de Filosofía y Humanidades, Santiago, Chile. Correo electrónico: sfalabella@ gmail.com ${ }^{2}$ Universidad de Chile, Facultad de Filosofía y Humanidades, Santiago, Chile. Correo electrónico: bernardita.domange@gmail.com
\end{abstract}

En el año 2007 llegó por primera vez a Chile el legado literario que Gabriela Mistral dejó al morir en Estados Unidos. Este hito histórico ocurrió 50 años después de la muerte de la autora en Nueva York. Específicamente, el Poema de Chile, libro que dejó inconcluso al morir, cobró nueva vida. Entre el material que llegó se encontraron un manuscrito y tres versiones adicionales al último manuscrito conocido. La importancia de este libro póstumo es que en él Mistral "piensa Chile". Es más, es la única obra de la autora en la que se elaboran de manera unitaria y sistemática las claves éticas, espirituales y estéticas de Chile. En el Poema de Chile se destacan valores democráticos y de valoración de elementos de subjetividades marginales a la lógica patriarcal y patrimonial históricamente imperantes en su tierra.

Palabras clave: Gabriela Mistral, género, legado, Poema de Chile, transición a la democracia.

For the first time in 2007 we received in Chile the full literary heritage of Gabriela Mistral from the United States. This historical benchmark is taking place 50 years after the author's death in New York. This is especially relevant for her posthumous book Poema de Chile, left unfinished upon the author's death. This recently arrived material contains one new manuscript and three new versions of the book, all different from the manuscript that was known until now. The importance of Poema de Chile is that it is the single work of Mistral in which the author takes on the unitary and systematic task of "thinking Chile". It is in this oevre that she puts forth her ethical, spiritual, aesthetical and political ideas in regard to her country. It is a book laden with utopian values and valuation of subjectivities of those traditionally marginalized by patriarchal and patrimonial logics.

Key words: Gabriela Mistral, gender identity, cultural heritage, Poema de Chile, transition to democracy.

\footnotetext{
* Este artículo presenta parte de los resultados del Proyecto FONDECYT Regular N 1085261 "Hacia una edición crítica del Poema de Chile: estudio genealógico y comparativo de los manuscritos de Gabriela Mistral y las versiones publicadas de la obra". 2008-2010.
} 


\section{INTRODUCCIÓN}

En diciembre del 2007 comenzamos a recibir en Chile con entusiasmo el legado mistraliano que, de acuerdo al testamento de la autora, luego de su muerte quedó en manos de su albacea, Doris Dana. Se trató de un esfuerzo conjunto en que participaron múltiples actores, en especial el Archivo del Escritor de la Biblioteca Nacional ${ }^{1}$. El arribo de este legado ocurrió cincuenta años después de la muerte de Gabriela Mistral (1889-1957) y permitió, por primera vez, el total acceso al valioso patrimonio de sus manuscritos, correspondencia, fotografías, objetos personales y hasta archivo de cine y audio, que la autora fue cuidadosamente acumulando durante los 35 años que vivió fuera de Chile ${ }^{2}$. Este es un importante hito en el escenario de la historia cultural de Chile: nunca antes habíamos contado con un contexto tan favorable para poder trabajar de manera simultánea e inmediata la obra y los archivos mistralianos. Gracias a este aporte podremos lograr un entendimiento más completo y sutil del complejo tejido en el que se constituyó la obra de una autora como Gabriela Mistral, su legado, simbólico y material ${ }^{3}$.

La llegada del legado de Mistral al país dio comienzo a una nueva etapa de interpretación y resignificación del legado de la autora, especialmente del Poema de Chile, obra inconclusa y cuyas ediciones posteriores presentan evidentes problemas (Falabella 1994, 1996, 2003) aún por resolver. Es más, es esta la obra en la que Mistral "piensa a Chile": elaborando de manera unitaria y sistemática claves éticas, espirituales, estéticas y políticas para Chile. El presente artículo da cuenta de parte de los resultados del Proyecto FONDECYT Regular N ${ }^{\circ} 1085261$ "Hacia una edición crítica del Poema de Chile: estudio genealógico y comparativo de los manuscritos de Gabriela Mistral y las versiones publicadas de la obra.", centrándose en el gran impacto que implicó la llegada del legado para el estudio de la obra Poema de Chile.

\footnotetext{
1 Entre ellos se encuentran el embajador de Chile en Estados Unidos, Mariano Fernández, quien fue el responsable de gestionar la llegada de las obras desde Estados Unidos; la directora de la DIBAM, Nivia Palma, quien se ocupó de dirigir la gestión de organización del corpus una vez arribado; la Ministra de Cultura, Paulina Urrutia, quien supervisó los trámites y gestiones para traer de vuelta el legado; Pedro Pablo Zegers, conservador del Archivo del Escritor, quien ha estado a cargo del estudio del material desde su llegada.

${ }^{2}$ La obra mistraliana anterior está recopilada por Pedro Pablo Zegers en Recopilación de la obra mistraliana 1902-1922. Este trabajo reúne según el editor, "dentro de lo posible, toda la producción literaria de Gabriela Mistral, entre los años 1902-1922. Es decir, desde sus primeros escritos juveniles, en Vicuña y La Serena, hasta aquellos trabajos que aparecen en diarios y revistas de 1922, fecha en que la poetisa deja el país, para ir a México, y sólo retornar por cortos periodos a su patria” (Zegers 2002: 17).

${ }^{3}$ El arribo de los manuscritos corresponde a un esfuerzo coordinado por los gobiernos de la Concertación de Partidos por la Democracia del Presidente Lagos y la Presidenta Bachelet, la primera mujer Presidenta de Chile. Así por ejemplo, la ex Ministra de la Cultura Paulina Urrutia defiende la gestión de la coalición: "Quiero desmentir versiones de que el Gobierno no ha hecho nada en torno a Gabriela Mistral. Gracias al ex presidente Ricardo Lagos se derogó el decreto ley 2.560, que impedía el cobro de los derechos de autor, y se trasladaron a Chile los restos de Yin Yin. Ahora, por mandato de Michelle Bachelet, estoy conversando con los albaceas." (El Mercurio 2007).
} 


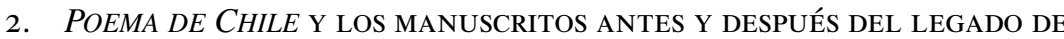 Gabriela Mistral}

"Antes de morir quiero caminar parcelas" Gabriela Mistral

Antes de la llegada del legado, los manuscritos que se conocían de Mistral estaban depositados en su gran mayoría en la Biblioteca del Congreso de los Estados Unidos y en la Biblioteca Nacional de Chile. De los papeles que estaban en la Biblioteca del Congreso norteamericano existía una versión microfilmada, también disponible en ambas bibliotecas. Este material ha sido de gran significancia para la edición y el estudio del Poema de Chile, dado que la elaboración de sus ediciones se basó en estos archivos. Sin embargo, con la llegada del legado en diciembre del 2007 no solo se amplió dramáticamente el corpus de material a ser revisado para estudiar la obra con un gran aporte de nuevo material manuscrito antes desconocido. A su vez, la heterogeneidad del contenido del legado se complejizó: se trata de un corpus multimediático que nos abre desconocidas posibilidades de acceder a nuevos sentidos de la obra.

Ya con anterioridad se ha mencionado lo amplio y complejo que es el estudio de Poema de Chile: se trata de una obra inconclusa de al menos 89 poemas que debían cumplir con la misión de, entre otros: "Contar en metáforas la largura de Chile, sus 3 climas, etc.” (Mistral Rollo 1: 298) ${ }^{4}$. Además, su elaboración contiene una gran cantidad de tiempo: es una "obra en marcha" que nunca termina, ya que su autora la reescribe continuamente. En efecto, Mistral comienza a elaborar esta obra al salir de Chile en 1922 y la deja inconclusa al morir. Sabemos por los manuscritos y varios testimonios que la autora le dedica a este libro intensamente sus últimos años, presintiendo su muerte 5 . En la medida que van pasando los años, cada poema fue registrando múltiples reescrituras y muchos quedaron sin terminar.

Con el Poema, Mistral busca hacer un gran lienzo poético de Chile: "Haciendo yo una especie de mapa medieval de Chile, me represento las regiones según ese estilo, personalizándolas en una bestia o en un cultivo" (Mistral 1957: 190) ${ }^{6}$. Se trata de una estructura alegórica y de contigüidad, esto es, de poemas encadenados, donde cada poema es un eslabón hilvanado por la geografía del territorio de norte a sur (Falabella 2003: 77 y 81).

\footnotetext{
${ }^{4}$ Todas las citas de poemas de Mistral corresponden a los manuscritos, especialmente a los del primer rollo del microfilm "Gabriela Mistral papers".

5 Hay variados elementos que documentan este hecho. Por ejemplo en las palabras "Al lector" de la edición del 1967 de Poema de Chile, Doris Dana señala:

Es necesario dar a conocer cómo llegó a publicarse este libro póstumo de Gabriela Mistral. Ella, al morir, dejó inconclusa la obra. Durante los últimos veinte años de su vida tuvo una preocupación continua: escribir poemas sobre toda suerte de asuntos relacionados con su país: cantar sus plantas, animales, los ríos, el mar, los lugares y sensibilizar los problemas del campesino y la reforma agraria; escribir para ella estos poemas no fue un afán literario sino una necesidad vital (Mistral 1967: s/p).

6 También en Tala, Gabriela Mistral explica la noción de recado, destacando su cercanía a la carta (testimonio y oralidad), a la poesía, a la tierra y a su loca poética, vinculada a la marginalidad y a su ser mujer (Mistral 1979: 161).
} 
En la siguiente cita de una carta podemos apreciar la forma en que el acto de escribir sobre Chile se convierte en un acto mágico, casi sagrado, de volver a su pasado de infancias y su tierra:

Con los años nos vamos reduciendo a escombro. ¡Cuánto temía esto yo cuando era una muchachita elquina que no se cansaba de trepar los peladeros buscando flores y piedras! Y cómo echo de menos los ojos de gavilán con que deletreaba las briznas más lejanas y hasta el temblor del pelaje de un conejo al otro lado del Valle. Tuve ese surco de surcos, mi Elqui patrio, más conocido para mí que mis versos o el mapa de mis manos, y me lo tuve por rebose de unos sentidos certeros y alertísimos. Nada de eso vuelve, queridos. Ahora, escribiendo estrofas de mi "Recado de Chile", huelo en el aire frío, atrapo el frescor de la nieve, un aroma llega roto por los pinares, y en el que reconozco, pobre de mí, las manzanillas que mi madre ataba para sus infusiones. Y me acude un aroma a brasero que es toda mi vida de maestrita pobre en escuela más pobre aún (Vargas Saavedra 1990: 229) 7 .

La escritura de su "Recado de Chile" conectaba a Mistral con la memoria nostálgica de su cuerpo joven y sano que la transportaba de vuelta a su juventud en el Valle de sus infancias. El resultado es que el proceso de elaboración que recoge la obra es altamente complejo, por lo que su constante reescritura implica que ésta vaya adquiriendo una multiplicidad de planos y simultaneidades, rindiéndola heterogénea (Falabella 2003: 51).

Sin embargo, esta riqueza juega en contra de la obra a la hora de ser póstumamente editada por Doris Dana, albacea de Mistral en 1967. Esta primera edición de Dana es diez años posterior a la muerte de Mistral y tiene como ciudad de origen Barcelona, marcando una vez más la "extranjería" de la obra de Mistral ${ }^{8}$. Es en base a este primer trabajo que todas las futuras ediciones fueron elaboradas (Falabella 2003: 49). Lo problemático de este hecho es que la edición de Pomaire contiene importantes errores, como se ha demostrado contrastando las ediciones con los manuscritos (Falabella 1994, 1996, 2003).

Es en el Archivo del Escritor de la Biblioteca Nacional de Chile donde está el corpus de manuscritos anteriores al año 22, que incluyen los papeles de su primer libro, Desolación (1922). La autora comenzó a viajar constantemente y sólo volvió a Chile por cortos periodos. En la medida que fue acumulando papeles, estos viajaron con ella. Gabriela Mistral siempre procuró ser acompañada por una serie de mujeres ayudantes. Su albacea Doris Dana ha sido la más conocida; sin embargo, no es la única que estuvo en el círculo íntimo de Mistral, ella misma señala en una entrevista que la autora siempre estuvo rodeada de gente que le ayudó con sus papeles: "En México, Consuelo Saleva. En Italia, Fabricini, una muchacha napolitana. En Nueva York había personas que venían a la casa a escribir cosas, pero no en forma permanente, sino part time" (El Mercurio 2002). Entre las personas que la ayudaron encontramos a su amiga Palma Guillén y la maestra argentina Marta Salotti.

Uno de los aprendizajes que se desprende del estudio sostenido de los manuscritos de la autora es que estos no son papeles al azar. La obra de recopilación, que tal vez sea caótica en su orden, es sistemática en el tiempo. La documentación de los

\footnotetext{
${ }^{7}$ El destacado en cursiva es mío.

${ }^{8}$ Al igual que Desolación, Tala y Ternura, Poema de Chile se publicó primero en el extranjero, este último sólo se publicó en 1996 en Chile. La única excepción es Lagar, que fue publicado en Santiago de Chile en 1954 por la Editorial del Pacífico.
} 
manuscritos abarca toda una vida de producción literaria: un pliegue privado respecto a la obra publicada. Esto es, una suerte de microcosmos de "otra" vida. Claramente Gabriela Mistral tiene desde un principio una preocupación consciente y sistemática por conservar sus papeles.

En 1965, ocho años después de la muerte de la autora, Magda Arce encuentra nueve baúles en la casa de Mistral de Santa Bárbara, California, Estados Unidos. Es razonable suponer que el contenido de estos baúles corresponde al material itinerante de la autora $(1922-1950)^{9}$. Otro grupo de material fue el que la autora dejó en su casa en Nueva York, donde pasó los últimos siete años de su vida. En definitiva, cuando Mistral murió (en 1957), sus manuscritos y otros materiales culturales habían quedado repartidos en tres lugares geográficos distintos: primero, está aquello que dejó en Chile; luego, los papeles de los baúles de Santa Bárbara, y finalmente el material que dejó en su casa de Nueva York. Después de su muerte, el gobierno de Chile hizo un gran esfuerzo por repatriar el cuerpo físico de Mistral, pero su "segundo cuerpo", su legado de manuscritos y otros objetos de relevancia para el estudio y valorización de la obra de la autora, quedaron mayoritariamente en EE.UU. bajo la responsabilidad de su albacea, Doris Dana.

Abrumada por la cantidad de material y la responsabilidad que estos conferían, Dana depositó lo más valioso en la Biblioteca del Congreso de Estados Unidos ${ }^{10}$. Fue en base a este material que Dana editó el Poema de Chile en 1967. Luego, entre los años 1981 y 1982, Dana también lideró el esfuerzo por hacer microfilms de los papeles manuscritos más destacados en relación a los libros de Mistral, gracias al auspicio del gobierno chileno y la OEA. A partir de 1982, este material quedó disponible al público tanto en la Biblioteca del Congreso de los Estados Unidos, como en la Biblioteca Nacional de Chile.

Al revisar estos microfilms, lo primero que llama la atención es que más de la mitad de este material está dedicado a Poema de Chile (Falabella 1994, 2003) ${ }^{11}$. La investigación del cuál parte da cuenta este escrito se basó en estos mismos microfilms, los únicos manuscritos del Poema de Chile accesibles en el momento de postular a dicho proyecto ${ }^{12}$.

Sin embargo, con la llegada del legado en diciembre del año 2007 se amplió de manera significativa el universo de material para acercarnos de manera sistemática y rigurosa a lo que Mistral tuvo en mente en cuanto a la historia, sentido y forma final de esta obra, incluyendo un criterio de crítica textual ${ }^{13}$.

\footnotetext{
${ }^{9}$ La profesora Magda Arce, en 1964 llegó desde Chile a la Universidad de California, Santa Bárbara, para ejercer como profesora visitante y en 1965 encontró nueve baúles con documentos inéditos de Gabriela Mistral en una casa que habitó la autora durante tres años en Santa Bárbara, Estados Unidos (Arce y von dem Bussche 1993).

10 Información recabada en una entrevista a Gastón von dem Bussche en 1996, hecha por Soledad Falabella Luco. En ella, von dem Bussche relató el abandono que sintió Doris Dana por parte del Estado de Chile.

11 Muy posiblemente esto tenga que ver en primer lugar con el hecho de que su proceso de elaboración comenzó cuando partió de Chile (en 1922) y, por tanto, abarca 35 años de creación.

12 Se sabía que había una intención por parte de la nueva albacea Doris Atkinson (Doris Dana murió el 2007) de donar el material, pero no estaba claro a qué institución se lo iba a donar, en qué país estaría dicha institución y menos cuándo ocurriría dicha donación.

13 A pesar de que el inventario oficial con todos los objetos que forman parte del legado aún no está disponible al público, sabemos que entre éstos se encuentran 562 piezas, incluyendo libros de su colección personal, fotografías, cartas, películas, cintas de audio, materiales inéditos de poesía y prosa, objetos personales, obras pictóricas, piezas ornamentales y de uso cotidiano. Parte de esto se puede ver en el portal de la DIBAM http://salamistral.salasvirtuales.cl:90/muestraLegado.php
} 
En diciembre de 2007 se anunció el comienzo de la llegada del material del legado de la autora y en marzo del año 2008 se dio inicio a la presente investigación ${ }^{14}$. Desde temprano contamos con acceso para estudiar el nuevo material en el Archivo del Escritor, apoyando la clasificación y catalogación de la correspondencia de Gabriela Mistral $^{15}$. El proceso de clasificación y catalogación del legado comenzó por los objetos y la correspondencia que ella recibió. Recién en el año 2009 pudimos acceder a la poesía. Así, los hallazgos relativos al Poema de Chile ocurrieron hacia fines de la investigación, cuando comenzamos a trabajar simultáneamente con el material de los microfilms y el recién llegado material del legado poético.

Así, nuestro proyecto de investigación se abrió al nuevo material e incorporó este vasto y desconocido universo a su campo de estudio. Como resultado, hallamos no una, sino múltiples versiones de la obra, cada una mecanografiada con diversas grafías y en un archivador propio. A partir de esto nos preguntamos: ¿acaso estos nuevos hallazgos significarían que ya no habría un manuscrito "arquetipo", madre, del libro? Esto es, ya no sabíamos si el primer manuscrito de los microfilms dejaba de primar sobre otros textos. Estos hallazgos son un ejemplo de cómo se complejizaron radicalmente las bases del planteamiento del proyecto, cuyo objetivo primordial era llevar a cabo un estudio textual, genealógico y comparativo de los manuscritos del Poema de Chile de Gabriela Mistral respecto a las versiones publicadas de la misma obra. A partir de ello se buscaba producir una versión según un paradigma de lectura crítico $^{16}$. Sin embargo, con la llegada del legado, la investigación ya no abarcaba sólo un manuscrito de Gabriela Mistral, sino que dos manuscritos y tres nuevas versiones que problematizaron la hipótesis de trabajo ${ }^{17}$. En el diseño de nuestro proyecto teníamos como elementos ejes para configurar una versión crítica la existencia de un manuscrito arquetipo (el manuscrito que aparece en el Rollo1 de los microfilms). También, los índices eran claves para ordenar y datar más adecuadamente el Poema. A continuación damos cuenta de los hallazgos respecto a estos tres temas eje de la investigación: índices, manuscritos y versiones y datación.

Como señalamos arriba, el hallazgo de índices fue una meta para guiarnos en la investigación. En los microfilms (seleccionados por Dana) había cuatro índices. Sin

\footnotetext{
${ }^{14}$ El temprano acceso se debe gracias a la generosidad para con la investigación de su Director Alfonso Calderón y Subdirector Pedro Pablo Zegers. Sin su confianza en nuestro equipo y proyecto, la investigación no habría avanzado en los hallazgos que se presentan en el presente artículo. Asimismo, Tomás Harris regaló su paciencia y demostró su tolerancia al cedernos su espacio de trabajo y poder así trabajar con los microfilms. Junto con agradecerles a ellos, agradecemos también a Jaime Nahum, que colaboró en el proyecto al coordinar la investigación con infinito compromiso.

15 El equipo de tesistas del Proyecto de investigación colaboró con la clasificación de la correspondencia. De esta forma se generó un temprano contacto con la paciencia, el oficio y la dedicación que se requieren para el trabajo con manuscritos.

${ }^{16}$ Parte clave de los objetivos secundarios del proyecto eran: transcribir la última versión del libro que dejó Gabriela Mistral, que forma parte de los Gabriela Mistral Papers; documentar e incorporar las últimas correcciones de Gabriela Mistral; datar el Poema de Chile; anotar las variantes de las ediciones existentes de la obra y documentar, esclarecer y corregir las discrepancias entre el manuscrito y las versiones publicadas. Asimismo, se estudiarán los modelos de ediciones críticas y definirá un estándar para el estudio.

17 Entendemos por manuscrito de la obra todo texto que contenga la grafía de la autora. Entendemos por versión de la obra todo texto de la obra que no presente la grafía de la autora. Así, los dos manuscritos del Poema de Chile de Mistral son un conjunto de poemas e índices mecanografiados y que presentan la grafía de la autora. En cambio, las tres versiones que encontramos del Poema de Chile no contienen grafía alguna de Mistral.
} 
embargo, estos índices no necesariamente reflejaban el orden final de los poemas, sino que Mistral los clasificaba muchas veces según su tipo de rima o su tema, como se ve a continuación:

\section{Rimas}

POEMA DE CHILE (copiadas a máquina en este cuaderno)

1. Hallazgo (eo)

(hay otro cuaderno)

2. Ruta (a a)

3. Desierto (e o) $\quad 3^{\mathrm{a}}$ - Golondrinas del yodo

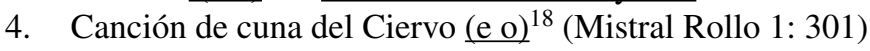

Con el material del legado se amplió el número de índices de cuatro a siete. En estos nuevos índices encontramos títulos de poemas desconocidos que no aparecen en el microfilm. Es importante tener en mente que no sabemos quién ordenó las piezas del legado, por lo que tampoco sabemos si estos nuevos poemas efectivamente corresponden a Poema de Chile ${ }^{19}$. Sin embargo, sí reconocemos parentesco entre estos nuevos índices y los índices anteriores. Ejemplo de estos poemas mencionados son: "El ciervo", "Encuentro del ciervo" y "Ronda de olores". También encontramos otros que luego pasaron a ser parte de Lagar, como "Altos hornos, Golondrinas del yodo", y otros que fueron publicados en antologías, "Salto del Laja" y "Volcán de Osorno". Además, estos nuevos índices contienen anotaciones señalando fechas de publicación de algunos poemas en revistas. Llama la atención que los poemas que encontramos en los índices exceden el número de poemas que hay en el manuscrito vinculado a dicho índice.

Al igual que los índices de los microfilms, los del legado están mecanografiados y contienen grafías de distintas personas. Así, vemos en el índice número 3 del que nosotros nombramos Archivador 1 el siguiente ejemplo:

\section{POEMA DE CHILE \\ $-4-$ \\ Perdiz $\quad(3,4,22) \checkmark$ \\ Piedra de amistad ( ) $\checkmark$ \\ "Qué será de Chile en el cielo" (1) \\ -Qué año o qué día moriste $\sqrt{ }(22)$ \\ $\begin{array}{lll}\text { Raíces } & (22) & \checkmark \\ \text { Reparto de Tierra } \checkmark & \text { [Aun vivimos] } & \\ & \text { (Mistral Rollo 1: 13). }\end{array}$}

Los índices están todos mecanografiados y, como señalamos arriba, no sabemos quién hizo la selección y ordenamiento de material que contienen. No sólo sería

\footnotetext{
18 Transcripción del microfilm. La letra cursiva corresponde a la grafía de Mistral; la subrayada a una de sus ayudantes. El resto está mecanografiado.

19 El legado ha pasado por múltiples manos, para comenzar, las de Doris Dana.

${ }^{20}$ La transcripción corresponde al manuscrito del Archivador 1. La letra subrayada corresponde posiblemente a una de sus ayudantes, mientras que la que se encuentra en negrita es la letra de Doris Dana. Agradecemos a Pedro Pablo Zegers por habernos facilitado textos manuscritos de Doris Dana para comprobar esta información. Este material es inédito.
} 
ingenuo pensar que Mistral es la única que estuvo en contacto con el material (y que, por lo tanto, se trata de material virgen), sino que estaríamos desconociendo evidencia histórica que señala lo contrario. Además de las múltiples manos que trabajaron sobre el Poema de Chile, el legado llegó a nosotros en material de audio y video, en el cual encontramos el siguiente segmento en el que Gabriela Mistral y Doris Dana revisan páginas de Poema de Chile, y discuten en torno a los índices de la obra:

GM: (...) Poema de Chile y aquí están varias cosas, ¿ah?... Esto es lo que tú has copiado, ¿ah?

DD: No. Es toda la cosa, copiada y no copiada. Si está copiada, está escrita aquí. ¿No? Por ejemplo, "Tacna" no está copiada,

GM: Bueno, pero hay una copia de esto. ¿Ah?

DD: Oh, sí, sí.

GM: ¡A ver! Vamos a ver lo que hay aquí de ese Poema de Chile. Tenemos que haber perdido cosas...

DD: Es una repetición, Gabriela. De esa primera cosa. Todo es repetición. Es un arreglo según el título.

GM: Bueno. ¿Y, por qué no haber copiado esas cosas?

DD: Porque el tiempo (sic)... Hay tiempo, ¿no? Y...

GM: Mira, aquí hay cosas que no las he visto yo en todo eso: "La desvelada"...

DD: Sí, está. Está aquí.

GM: "La caída de Europa"...

DD: Sí, todas esas cosas están. Pero, no están en Poema de Chile, ¿no?

GM: ¡Ah, pero la cuestión es que estas cosas estén con los textos! Están solo los nombres, ¿no?

DD: No. No, los textos están en otro libro. Este es el libro índice nada más.

GM: En este libro yo creo que cabe bastante, ¿no? ¿Como para...? ¡A qué tenerlo a lo loco! Así, ¡sin sostén!

DD: ¿A lo loco?

GM: Sí. A lo loco... Sin sostén.

DD: ¿Qué es eso de sin sostén?

GM: Pues, copiar la materia y ponerla aquí. ¿O vas a hacer un cuaderno de títulos? ¡Eso no se ha visto nunca!

DD: (ríe) Bueno, pondremos más cosas...

(Silencio, se escucha que Gabriela revisa)

GM: Ese "Cuco”. ¡No lo he visto copiado nunca parece...!

DD: Sí. Está copiado.

GM: Y ese de "Cuando voy al Valle del Elqui". También no lo he visto nunca.

DD: ¿Dices que nunca ha (sic) leído el "Cuco”? Y lo leyó (sic) ayer.

GM: Sí, sí. Tengo un vago recuerdo. Pero, es una poesía que siempre se me... Que yo debí alargarla, me gusta mucho, ese tema de la jugarreta...

DD: ¡Ah! ¿Y, Gabriela, esta cosa de "Flores” que no vimos antes? (Silencio) Yo creo que es una cosa reciente, ¿no? Porque está escrito en este papel.

GM: No. Es del Poema de Chile, es "Nadie se allega al fantasma". Y si no lo sacas luego... ¡Adiós! Porque está muy borroso. (...)” (Mistral Track 2).

La transcripción nos permite ser testigos de una escena como muchas que deben haber ocurrido. El hecho que las hayan grabado es significativo, debe ser característica de la forma de trabajar juntas que tenían Doris Dana y Gabriela Mistral. De la cinta se desprende que Doris Dana elaboraba ella misma índices, y que estos índices no 
concuerdan con lo esperado y necesitado por Mistral. La autora insiste en que estos no tienen sentido para ella, señalando que están elaborados "a lo loco" y "sin sostén":

DD: ¿A lo loco?

GM: Sí, a lo loco... sin sostén.

DD: ¿Qué es eso de sin sostén?

GM: Pues, copiar la materia y ponerla aquí. ¿O vas a hacer un cuaderno de títulos?, ¡Eso no se ha visto nunca!

Resulta evidente que la lógica de orden de Doris Dana no concordaba con la necesidad de Gabriela Mistral, la que se muestra molesta e irónica. Lo que reclamaba la autora era trabajar directamente sobre la materia poética misma. Además, es notorio el grado de desconocimiento del español de Doris Dana y cómo esta falta de familiaridad impacta en su capacidad de captar las sutilezas del lenguaje de Mistral. No entiende cuando Mistral usa un lenguaje figurativo y palabras menos cotidianas.

Junto con ello, es significativo que, a partir de esta grabación, tanto la autoría como la legitimidad de los índices que nos encontramos en los manuscritos deban ser cuestionadas. Claramente, algunos fueron elaborados por Doris Dana, aunque no nos consta que ella los haya elaborado todos. También sabemos que éstos no concuerdan con la lógica de trabajo de Mistral, y que mezclan poemas de distintos libros, lo que enoja a la poeta.

Asimismo, cabe también preguntarse si alguna vez Mistral misma ordenó el Poema de Chile como libro. Su modo de trabajo, que se puede deducir de los manuscritos, da cuenta de una intensa labor de creación poética que se mantenía durante mucho tiempo abierta a correcciones. La selección de poemas para armar libros era un proceso posterior, de modo que tomaban forma de una manera más abstracta, casi arquetípica, a nivel del pensamiento de la autora. Ella no reclama índices, pide trabajo con la transcripción de los poemas, los que ella sabe que se están borrando a raíz de que fueron escritos con lápiz mina (grafito). Su preocupación es material. Todavía no está armando el "índice" del libro llamado Poema de Chile.

Otro de los ejes del proyecto de investigación que se vio fuertemente impactado por la llegada del legado fue el hallazgo de otras cuatro versiones del Poema de Chile: en el material que llegó encontramos cuatro archivadores, cada uno con una distinta versión de la obra. En total tenemos cinco versiones distintas del Poema.

Los cuatro archivadores se fueron enumerando a medida que se encontraban. El archivador 1 contiene el manuscrito más completo, semejante al manuscrito del Rollo $1^{21}$. Sin embargo, hallamos índices que no aparecen en el microfilm. Este material señala la existencia de poemas que no se encuentran en los otros manuscritos del Poema de Chile. ¿Serán estos los índices que se discuten en la grabación antes citada? Además, es el único archivador donde participa la grafía de Mistral (presenta en total cuatro grafías distintas).

El archivador 2 no presenta correcciones manuscritas por parte de la autora, pero sí presenta escasamente intervenciones de una grafía que posiblemente puede ser de

\footnotetext{
21 El archivador 1 tanto en su índice como en los poemas tiene varias grafías participantes. Dentro de las que podemos distinguir se encuentran la de Mistral y la de Doris Dana. Este archivador presenta viñetas que dicen "Indice", "Comienzo", "La ruta", "Intercalar", "Sacadas".
} 
Marta Salotti o Margaret Bates. Asimismo, este archivador contiene menos poemas que el archivador $1^{22}$. Luego, el archivador $3^{23}$ es la versión final que se presenta para publicar en la editorial Pomaire en 1967; esta versión tiene tres poemas menos que el archivador 2 y 4 -"Tacna", "Cinco tiempos del huemul”, "Cielo estrellado (igual a "Noche andina", cambia solo el título)-, está más acotado, más intervenido o más "terminado". Finalmente, el archivador 4 contiene un índice anotado por Alone y un corpus de poesía corregido por Doris Dana ${ }^{24}$. En este cuarto manuscrito vemos los mismos poemas que encontramos en el archivador 2, exceptuando "Desierto".

\title{
3. Importancia y Claves del PoEma de Chile
}

\author{
Mientras te hablo, mira mira \\ reparten tierra y huertas \\ Oye los gritos, los “ivivas!" \\ el alboroto, la fiesta \\ ¿te das cuenta?; Entiende, mira! \\ Es que reparten la tierra. \\ Gabriela Mistral
}

El impulso que da origen a la preocupación de Mistral por escribir un texto sobre Chile se deja ver por primera vez en sus manuscritos en 1922 (Falabella 1994: 22; 2003: 29, 57, 124), año en que la autora sale de Chile por primera vez para trabajar en el gobierno de la revolución mexicana, para hacerse cargo de la fundación del sistema de escuelas rurales para la nueva nación: "Cuando Gabriela Mistral llegó a México en 1922, José Vasconcelos había echado a andar la gran máquina de la Secretaría de Educación -poca herramienta, en aquellos años, y mucho espíritu- y todos los jóvenes de entonces íbamos con él llenos de entusiasmo" (Mistral 1997: V). Mistral seguirá escribiendo su preocupación por Chile, dando así forma a una obra que lentamente irá encontrando su propio "cuerpo".

Esta obra tiene su comienzo como un Recado que ella le deja a los suyos (propios). El Recado es un género propio de Mistral, que define así en una nota, al final Tala:

\section{"Recados"}

Las cartas que van para muy lejos y que se escriben cada tres o cinco años, suelen aventar lo demasiado temporal -la semana, el año- y lo demasiado menudo -el natalicio, el

\footnotetext{
22 En este segundo archivador: "Balada de la menta", "Canción del buen sueño", "Canción de cuna del huemul", "Desierto", "Misioneros", "El valle central", "Otra versión de hallazgo", "Otra versión de Valle de Elqui", "Otra versión de la ruta", "Otra versión de animales".

23 El archivador 3 tiene el mismo contenido de poemas que la edición de 1967 y está ordenado en el archivador de igual forma como aparece en el libro publicado. Este archivador se encuentra prácticamente sin correcciones manuscritas ni de la autora ni intervenciones de otras grafías. Se presume que tiene menos trabajo que el archivador 2, pues no consta del mismo cuerpo textual y tiene menos correcciones que este. Faltan en este manuscrito los mismos poemas que en el archivador 2 y además: "Tacna", "Cinco tiempos del huemul", "Cielo estrellado (igual a "Noche andina", cambia solo el título).

${ }^{24}$ El índice del archivador 4 está revisado por Alone y Doris Dana y no se encuentra grafía mistraliana en ninguna parte de éste. La primera página de cada poema tiene un encabezado que dice: "Recado de Chile". Posee el mismo corpus del archivador 2, pero ordenados de distinta manera.
} 
año nuevo, el cambio de casa-. Y cuando, además, se las escribe sobre el rescoldo de una poesía, sintiendo todavía en el aire el revoloteo de un ritmo sólo a medias roto y algunas rimas de esas que llamé entrometidas, en tal caso, la carta se vuelve esta cosa juguetona, tirada aquí y allá por el verso y por la prosa que se la disputan.

Por otra parte, la persona nacional con quien se vivió (personas son siempre para mí los países) a cada rato se pone delante del destinatario y a trechos lo desplaza. Un paisaje de huertos o de caña o de cafetal, tapa de un golpe la cara del amigo al que sonreíamos; un cerro suele cubrir la casa que estábamos mirando y por cuya puerta la carta va a entrar llevando su manojo de noticias.

Me ha pasado esto muchas veces. No doy por novedad tales caprichos o jugarretas: otros las han hecho y, con más pudor que yo, se las guardaron. Yo las dejo en los suburbios del libro, "fuora dei muri", como corresponde a su clase un poco plebeya o tercerona. Las incorporo por una razón atrabiliaria, es decir, por una loca razón, como son las razones de las mujeres: al cabo estos Recados llevan el tono más mío, el más frecuente, mi dejo rural en el que he vivido y en el que me voy a morir. (Mistral 1979: 161).

Para Gabriela Mistral los Recados son una textualidad mediante la cual se toma contacto con la gente y la tierra. Ambos cobran vida en este género que tiene marcadas características estéticas, éticas y espirituales: vinculados a la tradición oral, lo pequeño y la humildad. La escritura de los Recados tiene un poder transformacional de proyectar a su autora en el tiempo y el espacio, hacia un lugar utópico y cargado de esperanza (Falabella 2003: 247-249). Además vemos aparecer claramente el elemento de la identidad de género, vinculado con aquello que es menor y está desplazado, una "clase un poco plebeya o tercerona", que legitima a partir de una razón "atrabiliaria" y "loca", como suelen ser las razones de mujeres, siguiendo a la autora. Es en esta obra donde escribe poemas cuyos nombres se vinculan con elementos espirituales, éticos y concretos a la vez, poemas como “¿Qué será de Chile en el Cielo?”25 "¿Adónde es que tú me llevas?" y "Reparto de tierra".

En el Poema de Chile Mistral escribe y reescribe su vuelta. Junto con ello, lleva a cabo un profundo trabajo para con la lengua, no solo revitalizándola, sino que ampliando su norma como veremos más adelante. Así comienza "Hallazgo", primer poema del libro:

\author{
Bajé por espacio y aires \\ y más aires, descendiendo, \\ sin llamado y con llamada \\ por la magia del deseo, \\ y a más que yo caminaba \\ era el descender más recto \\ y era mi gozo más vivo \\ y mi adivinar más cierto, \\ y arribó como la flecha \\ éste mi segundo cuerpo \\ en el punto en que comienzan \\ Patria y Madre que me dieron. (Mistral Rollo 1: 1)
}

25 De este poema solo se encuentra el título. 
El almita de Mistral, "éste mi segundo cuerpo", baja a la tierra chilena llamado por el grito de una madre que muere y también por su propio deseo: "sin llamado y con llamada / por la magia del deseo". El almita/fantasma "vuelve" a su tierra natal, "en el punto que comienzan/ Patria y Madre que me dieron"; tal como la autora se refiere a su tierra de origen. De esta forma, no solamente tenemos una revitalización de la norma (Mistral le infunde nueva vida al uso normal de las palabras), sino que una ampliación de la norma para hablar de su tierra: ya no le basta hablar de la patria tradicional (patria viene de pater, padre en latín), sino de una nueva noción, "Patria y Madre que me dieron". Junto con ello, Mistral abre la norma tradicionalmente masculinista vinculada al pater, al incluir a la madre, una madre con mayúscula que se equipara al padre. Con este gesto, junto con darle un nuevo uso a las palabras, las une de tal forma que la tierra a la que baja no puede ser contenida por un solo concepto, sino por una colaboración de dos: padre y madre ${ }^{26}$. Algo similar a lo que plantea la autora en su recado "Menos cóndor y más huemul", donde señala su preferencia por el huemul de la heráldica nacional, que para ella "quiere decir la sensibilidad de una raza: sentidos finos, inteligencia vigilante. Y todo eso es defensa, espolones invisibles, pero eficaces del espíritu." Contrapone a este ciervo nativo al cóndor, "hermoso buitre", sin embargo, se trata de una contraposición dialéctica, donde el énfasis en los valores del huemul finalmente generan una nueva interpretación del escudo de Chile:

Tal vez el símbolo fuera demasiado femenino si quedara reducirlo al huemul, y no sirviera, por unilateral, para expresión de un pueblo. Pero, en este caso, que el huemul sea como el primer plano de nuestro espíritu, como nuestro pulso natural, y que el otro lado sea el latido de la urgencia. Pacíficos de toda paz en los buenos días, suaves de semblante, de palabra y de pensamiento, y cóndores solamente para volar sobre el despeñadero del gran peligro (Mistral 1995: 40).

Este verso implica "otra" lógica: inclusiva y contigua, más horizontal. Los que tradicionalmente son vistos como opuestos, padre/madre, colaboran para constituir un todo más amplio y diverso, Mistral enuncia una tierra más democrática, pacífica, espiritual y equitativa.

También, con el uso de mayúscula en "Patria y Madre" la autora le da a palabras de uso común -padre y madre- un significado de trascendencia utópica. Siguiendo a Ernst Bloch, quien estudia la utopía y la esperanza en la cultura de los países, especialmente en su literatura, en Poema de Chile la función utópica está doblemente

\footnotetext{
${ }^{26}$ Es interesante notar que al poner un elemento masculino y uno femenino para denotar su tierra está haciendo algo distinto a lo que el crítico Grínor Rojo señala que la autora hace en "Nocturno de la consumación", poema del libro Tala (1938), donde se privilegia la condición femenina:

Entre este mundo y el otro, su punto de mira deja de ser en el "Nocturno de consumación" el amor por El Padre y pasa a ser una atracción por el silencio de La Tierra. Ser tierra, "quedarse con" la tierra, participar "mano a mano" de su destino heterodoxo, vis-á-vis los preceptos de la religión y la cultura establecidas, se transforma en el nuevo ideal. Es bastante evidente, y por lo mismo no creo que sea necesario insistir sobre la pertinencia de mi planteo, que lo que aquí se ha producido es tanto un relevo como una rejerarquización. El espectáculo mistraliano adquiere por lo pronto un carácter que no coincide con las versiones al uso de su religiosidad. (1997: 170).

Si en el "Nocturno de la consumación" se glorifica lo femenino, en "Hallazgo" de Poema de Chile, poema umbral que da el tono de apertura de canto y que por lo tanto es la llave musical que debe guiar la "escucha" del Poema, hay un fino trabajo de búsqueda de armonía. En este sentido, la utopía del Poema de Chile es una utopía más amplia, donde tanto lo masculino como lo femenino tienen un espacio validado.
} 
presente. Esto es, aquello que "propaga [el excedente cultural de la obra] hacia adelante y como una solución que proviene hacia nosotros desde el futuro y no desde el pasado" (Bloch 1988: 117), lo encontramos en el Poema de Chile, tanto a nivel del mundo enunciado como de la enunciación (Falabella 2003: 80) ${ }^{27}$. Así, el contenido del Poema, los elementos que nombra, no abarcan solamente el pasado en forma de memoria, sino que también contienen elementos del futuro, abren el texto con esperanza. Un ejemplo de ello es la tierra adónde el fantasma de Mistral lleva al niño. Así, anuncia en el poema "¿Adónde es que tú me llevas?”: "donde al mirarte la cara/ no te digan como nombre/ lo de "indio pata rajada",/ sino que te den parcela/ muy medida y muy contada" (Falabella 2003: 123).

En cuanto a la enunciación, esto es cómo se dice, podemos reconocer el valor de la tradición oral en el Poema. Así, hallamos un impulso hacia el futuro lleno de esperanza en la profecía de plenitud de la mama: "Ya me llama el que es mi Dueño." en el poema "Despedida" (84), que canta la culminación de la gesta del fantasma. Es más, a nivel de equidad de género, es importante tener en mente que la legitimidad de una mujer para hablar en público y tomarse la palabra no era la misma que hoy en día gozamos las mujeres en Chile. De hecho, durante su vida Gabriela Mistral no gozó de los mismos derechos sociales, culturales y políticos que los hombres ${ }^{28}$.

En Poema de Chile canto, sabiduría y memoria, capacidad de redención de sí misma y del niño y justicia social se aglutinan en torno a una ética y estética que tienen un sustrato espiritual en el que la fe y la magia de la palabra cargada de futuro están íntimamente relacionadas. Así, la enunciación del Poema es el canto que redime: sus palabras salvan. Poema de Chile se sostiene sobre una espiritualidad que le da razón de ser a la autora como salvadora. Al escribir Mistral no sólo redime al niño, sino también se redime a sí misma y a través de su canto redime "Padre y Madre que me dieron.”, esto es, su tierra. Con ello la autora está creando una obra dentro de una corriente universal de obra artística utópica, muy en boga en su tiempo ${ }^{29}$. Así, la autora está en diálogo con el pensamiento más progresista de su época, tales como el movimiento antibélico, las cartas fundamentales de la protección de los Derechos Humanos y los Derechos de los Niños, el sufragio de las mujeres, la Reforma Agraria y una espiritualidad ecuménica capaz de abarcar desde sus raíces campesinas, pasando por la Teosofía, la Antroposofía, el Budismo y el Cristianismo (Taylor 1975; Carrasco 1977 y Rojo 1997). De esta forma, el Poema de Chile está en concordancia

27 La traducción es nuestra. Bloch desarrolla este concepto a partir de un comentario hecho por Marx: Marx made an interesting comment to Ruge in 1843 about the relation of true ideology to the anticipation within the false consciousness of the former ideology that is not entirely false: "Our slogan has to be: the reform of consciousness not by dogmas but by analyzing the mystical consciousness that is still vague about itself. Then it will become clear that since long ago the world has the dream of a thing and the world only has to have consciousness to really possess the thing. It will become clear that it is not a matter of a great connecting line between the past and the future but a matter of fulfillment of the thoughts of the past." (1988: 117).

28 Recordemos que en el Chile de principios de siglo XX la relación de desigualdad era marcada: Las mujeres seguían estando legalmente subordinadas a los hombres, y los roles tradicionales que designaban el hogar como el lugar de la mujer persistían profundamente [en el imaginario social]. Por ejemplo, las mujeres casadas no tenían poder sobre su propiedad (...) fue sólo en 1925, cuando se abolieron algunas restricciones legales en contra de las mujeres, cuando se permitió que las mujeres testificaran en la corte. (Antezana-Pernet 1994: 168).

29 Agradecemos este importante comentario a Alejandra Del Río, quien además resaltó la importancia del pensamiento de Bloch respecto a las tendencias antibélicas de la postguerra. 
con el pensamiento de la postguerra y bien se podría leer como un mapa utópico de la geografía humana chilena.

\section{Conclusión}

Poema de Chile entrega claves para reflexionar críticamente respecto a temas éticos, estéticos, espirituales y políticos del país. Se trata de una obra utópica por la primera Premio Nobel en poesía en lengua hispana que canta mediante la voz de una almita-fantasma la esperanza, sin dejar de lado temas fundamentales e incómodos, para pensar la identidad y la justicia social en Chile. Se toma la palabra para promover lógicas “otras", heterogéneas. De esta forma construye resistencias que subvierten a la lógica patriarcal y patrimonial históricamente imperante en Chile, creando una escritura incluyente de aspectos masculinos y femeninos a la vez, como cuando para nombrar su tierra dice "Padre y Madre que me dieron." Así, ya desde su poema umbral "Hallazgo", nos enteramos que estamos ante el deseo de bajar a la tierra y recorrer "la largura de Chile" junto a una mama fantasma y un niño atacameño, ambos sujetos carentes de un lugar social de prestigio, que a pie recorren el territorio de norte a sur. Esto es, en esta obra se trata de un bajar hacia el "sur", una opción por el subdesarrollo, en cuanto a la posibilidad de armar otro desarrollo, donde se materialice su utopía ${ }^{30}$.

De manera análoga al almita del Poema de Chile, desde el año 2007 en Chile hemos presenciado el vertiginoso proceso de la "bajada" de otro "segundo cuerpo" de Gabriela Mistral. La investigación de los manuscritos de Poema de Chile de la cual da cuenta en parte este artículo se vio beneficiada y radicalmente impactada por el "hallazgo" de nuevos manuscritos, nuevos índices y otro material, como grabaciones de audio, que forman parte del legado. En este sentido, es importante subrayar el hecho de que junto con ampliarse el material en cuanto a cantidad, a la vez se hizo más complejo: hay nuevos elementos que entraron en juego y otro tipo de decisiones que tomar en relación a la crítica textual. Sólo después de un sistemático y riguroso trabajo de revisión de todo el material del legado se puede abordar de manera sólida Poema de Chile, el más sistemático e íntimo esfuerzo de nuestra Premio Nobel de pensar Chile. Es en esta obra en la que, junto con temas de clase, género y raza, aparecen los incómodos temas de la pobreza, de la tenencia de la tierra y los derechos de las personas marginadas del poder, todos estos aún no resueltos en la cultura de la transición a la democracia chilena. Queda entonces por delante el trabajo que permita la integración de este nuevo material con el fin de contar con una versión crítica de la obra. Sólo así podremos hacernos cargo con propiedad del Poema de Chile, y escuchar a su autora.

\footnotetext{
${ }^{30}$ Recuerda Ciro Alegría cómo ella hablaba con fruición de sus orígenes humildes y una genealogía mestiza: "Vengo de esa clase media que linda con el pueblo por pobreza." o "Pertenezco a esa mitad segundona y pobre de la clase media que está asimilada al pueblo por su ruina". Hablaba de "mi abuelo indio", abuelo que se mata de hambre y sed en la cordillera, arrepentido de haberle pegado a su mujer y de su abuela "judaizante". (1968: 28 y 43).
} 


\section{OBRAS CITADAS}

Alegría, Ciro. 1968. Gabriela Mistral intima. Lima: Editorial Universo.

Antezana-Pernet, Corinne. 1994. "Peace in the world and democracy at home. The Chileanwomen's movement in the 1940's". Latin America in the 1940's War and postwar transitions, ed. David Rock. Berkeley and Los Angeles: University of California Press.

Arce, Magda y Gastón von dem Bussche. 1993. Proyecto de preservación y difusión del legado literario de Gabriel Mistral. Santiago: Zig-Zag.

Bloch, Ernst. 1988. The utopian function of art and literature: selected essays. Trad. Jack Zipes y Franck Mecklenburg. Cambridge: MIT Press.

Carrasco, Iván. 1977. "El mito de Orfeo y el Poema de Chile de Gabriela Mistral", Revista Chilena de Literatura 9-10: 21-40.

Falabella, Soledad. 1994. “¿Qué será de Chile en el Cielo?” Propuesta de lectura de Poema de Chile de Gabriela Mistral. Tesis para optar al grado de Licenciado en Humanidades con mención en Lengua y Literatura Hispánica. Universidad de Chile. Facultad de Filosofía y Humanidades.

. 1996. “¿Qué será de Chile en el Cielo?" Propuesta de lectura de Poema de Chile de Gabriela Mistral", Mapocho Revista de Humanidades y Ciencias Sociales, [División de Bibliotecas y Archivos, Biblioteca Nacional, Santiago] 39:71-78.

—. 2003. ¿Qué será de Chile en el Cielo? Poema de Chile de Gabriela Mistral. Santiago: Coedición Universidad Alberto Hurtado y Ediciones LOM.

El Mercurio. 2007. "Los originales podrían estar un tiempo en la Biblioteca Nacional". Santiago. [http://diario.elmercurio.cl/detalle/index.asp?id=\{e02eb16e-4672-41e6-b222925bd906187c $\}]$

Mistral, Gabriela. Manuscritos, rollo 1, cuaderno 1, Archivo del Escritor, Biblioteca Nacional de Chile.

—. Manuscritos, Archivadores. Archivo del Escritor, Biblioteca Nacional de Chile.

- 1957. Recados contando a Chile. Santiago: Editorial del Pacifico.

1967. Poema de Chile. Barcelona: Editorial Pomaire.

1979. Tala. Santiago: Editorial Andrés Bello

1995. Gabriela Mistral. Escritos políticos. $2^{\mathrm{a}}$ ed. Santiago: Fondo de Cultura Económica

—. 1997. Lectura para mujeres. $8^{\mathrm{a}}$ ed. México: Editorial Porrúa.

2009. De viva voz. Track 1. Biblioteca Nacional de Chile.

Rojo, Grínor. 1997. Dirán que está en la gloria... (Mistral). Santiago: Fondo de Cultura Económica.

Taylor, C. Martin. 1975. Sensibilidad religiosa de Gabriela Mistral. Madrid: Gredos.

Vargas Saavedra, Luis. 1990. Tan de usted. Epistolario de Gabriela Mistral con Alfonso Reyes. Santiago: Ed. Universidad Católica de Chile.

Zegers, Pedro Pablo. 2002. Recopilación de la obra mistraliana 1902-1922. Santiago: Ril editores. 
